\title{
Level 8: Das ultimative Nachwort
}

Nun sind wir am Ende unserer Reise angekommen. Wenn Sie das Buch aufmerksam gelesen haben, verfügen Sie über eine gute Grundlage, um mit Ihrer Bibliothek zu einer Gaming-Bibliothek zu werden. Wie bereits am Anfang dieses Buches erwähnt, reichen selbst fast 200 Seiten nicht aus, um das Thema „Gaming in Bibliotheken“ abschließend zu beschreiben. Ich habe versucht, Ihnen vor allem viele Ideen und Gedanken zu präsentieren. Es ging mir nicht darum, einen Ist-Zustand in Bibliotheken zu beschreiben. Aus diesem Grund habe ich auch auf Best-Practice-Beispiele verzichtet.

Ja, es gibt wundervolle Beispiele für tolle Gaming-Aktivitäten in Bibliotheken. Und auf meinem Blog und in der Facebook-Gruppe „games4culture“ wird immer wieder darüber berichtet werden. Auf den beiden Plattformen finden Sie auch den Link zur „Gaming-Bibliotheks-Museums-Archiv-Landkarte“. Es handelt sich um eine GoogleMap, die ich im Februar 2014 gestartet habe und auf der sich alle Bibliotheken, Museen und Archive eintragen können, die etwas mit Gaming machen. Deshalb meinen herzliche Bitte: Machen Sie mit und füllen Sie diese Landkarte mit Ihren Aktivitäten.

Denken Sie immer daran: Sie können noch so viele Bücher zum Thema Gaming lesen, wenn Sie nicht selbst spielen, werden Sie nicht erfolgreich sein können.

Das Wichtigste aber ist, dass Sie verstehen, dass es beim Thema Gaming um ein eigenständiges Thema geht. Gaming ist kein Thema, um vermeintlich „buchferne“ Schichten für das Lesen von Büchern zu gewinnen. Games sind genauso relevant wie Bücher. Es gibt gute Bücher und es gibt schlechte Bücher. Es gibt gute Games und es gibt schlechte Games. Wenn Sie sich länger mit dem Thema beschäftigen, werden Sie merken, dass es innerhalb der Gaming-Kultur viele spannende und zugleich kontroverse Diskussionen zum Thema gibt. Diese Diskussionen drehen sich nicht nur um die Frage von Gewaltdarstellungen, Suchtverhalten, Medienschelte etc. Immer mehr Spieler kritisieren bestimmte Geschäftsmodelle. Immer mehr Spieler möchten „bessere" Spiele weitab vom Mainstream spielen.

Ich habe es schon sehr oft erwähnt: Für Bibliotheken bedeutet Gaming die größte Chance und die größte Herausforderung zugleich. Games waren nie eine Konkurrenz der Bücher, aber sie haben das Potential noch bedeutender als Bücher zu werden. Ich bin der festen Überzeugung, dass Gaming unsere Gesellschaft nachhaltig verändern wird.

Gamer würden nie auf die Idee kommen, Bücher zu kritisieren. Sie sehen keine Konkurrenz zur Welt der Games - im Gegenteil, viele lesen genauso gerne wie sie spielen. Geben Sie den Games und den Gamern eine Chance. Und leben Sie damit, dass Ihre Kunden in der Regel mehr vom Thema verstehen als Sie J

Dieses Buch ist eine spannende Aufgabe gewesen. Und es wäre nicht möglich gewesen, es zu schreiben, ohne die Mithilfe von vielen spannenden Menschen. Ich weiß, dass eine Aufzählung immer die Gefahr birgt, dass man jemanden vergisst. Trotzdem möchte ich mich bedanken und bitte jeden Menschen um Entschuldigung, den ich vielleicht hier vergessen habe.

Zuerst möchte ich mich bei meiner Familie bedanken. Sie haben mir immer wieder geholfen und mich motiviert.

Dann möchte ich mich bei Gabriele Fahrenkrog und Tanja Müller bedanken, die das Buch schon in der Frühform gelesen und mich beraten haben.

Ein besonderer Dank gilt auch Kelvin Autenrieth, der mit sehr vielen Tipps und Ideen geholfen hat. Er ist nebenbei Erfinder von „Bibcraft“ und natürlich ist er mein geschätzter Kollege bei „games4culture“.

Eine sehr große Hilfe - schon seit vielen Jahren - ist Martin Lorber von Electronic Arts. Auch hier ein ganz großes Dankeschön, nicht nur für die Fotos aus Games, 
welche ich in diesem Buch verwende, sondern auch für die vielen Kontakte und Anregungen.

Vielen Dank an das Team des Vereins Zukunftswerkstatt Kultur- und Wissensvermittlung e.V.

Danke auch an die Goethe-Institute in Ost-Asien, die es mir ermöglicht haben, in Ost-Asien zu recherchieren.

Ganz herzlichen Dank auch an Julia Bergmann, die mich überhaupt erst zu diesem Buch inspiriert und den Kontakt zum Verlag hergestellt hat.

Und natürlich gilt mein Dank auch dem deGruyter-Verlag und ganz besonders Claudia Heyer, die mich mit sehr viel Geduld und Verständnis begleitet hat - auch als klar war, dass wir eine sehr lange Verzögerung bekommen.

Herzlichen Dank an Eli Neiburger von der Ann Arbor District Library, der mit seinen Ideen und seinen Vorträgen eine unglaubliche Inspiration war und der mir gezeigt hat, dass Gaming die Bibliothek wirklich verändern kann.

Aber mein größter Dank geht an die Gamer, Games-Entwickler, Games-Researcher etc. da draußen, die die Gaming-Kultur sind. Teil dieser Welt sein zu dürfen, ist etwas Großartiges. 


\section{Level 9: Quellen und Links}

Kommen wir nun zum wirklich letzten Level des Buches. Ich möchte Ihnen noch ein paar Quellen und Links zur Verfügung stellen, damit Sie noch weiter in das Thema eintauchen können:

Beginnen wir mit Büchern, die Sie unbedingt lesen sollten:

Steven Johnson:

„Neue Intelligenz: Warum wir durch Computerspiele und TV klüger werden.“

Dieses Buch ist für Mitarbeiter, Kunden und Pädagogen interessant. Es ist ein erster Einstieg in das Thema und es hilft, Zusammenhänge zu verstehen.

Jane McGonigal:

„Besser als die Wirklichkeit! Warum wir von Computerspielen profitieren und wie sie die Welt verändern.“

Dieses Buch ist ein weiteres Standartwerk. Jane McGonigal beschreibt, was Computerspiele sind, warum so viele Menschen sie spielen und was wir von den Games lernen können. Unbedingt lesen!

James Paul Gee:

"What Video Games Have to Teach Us About Learning and Literacy."

Wenn Sie noch tiefer in das Thema eintauchen möchten und auch aus Sicht der Pädagogik mehr wissen möchten, dann sollten Sie dieses Buch unbedingt lesen.

Eli Neiburger:

„Gamers... In the Library?!”

Das Buch ist schon etwas älter, aber es zeigt ganz genau auf, was Bibliotheken im Bereich Gaming machen können. Eli Neiburger ist der Experte für Gaming in Bibliotheken. Sein Fokus liegt dabei auf Wettbewerben. Und wie man einen solchen Wettbewerb konstruiert, ist ebenfalls in dem Buch beschrieben.

Marc Prensky:

"Digital Game-Based Learning."

Wenn Sie mehr über das Thema "Lernen und Games" wissen wollen, dann ist dieses Buch meine erste Empfehlung. Es ist sehr gut geschrieben und zeigt, wie sich durch Gaming neue Wege des Lernens ergeben können.

Es gibt natürlich sehr viele weitere Bücher und Aufsätze zu diesem Themenkomplex. Aber die hier genannten Werke stellen einen sehr guten Einstieg dar.

Kommen wir nun zu Onlineangeboten, Communities, Blogs, Wikis und Webseiten:

Eine spannende deutsche Community ist die Facebook-Gruppe „games4culture“. In dieser Gruppe treffen sich alle Menschen, die sich mit dem Thema Gaming in Bibliotheken, Museen und Archiven beschäftigen möchten.

Weitere Informationen und Update zu diesem Buch und weiteren Projekten finden Sie auf meinem Blog www.christoph-deeg.de und auf www.games4culture.de. 
Die Initiative „Creative Gaming“ führt eine Vielzahl an spannenden Gaming-Projekten durch: http://creative-gaming.eu/

Die Computerspielschule in Leipzig lässt junge Gamer Spiele testen und bewerten: http://www.uni-leipzig.de/ compsp/Csl/

Gleiches gilt für die Computerspielschule in Greifswald, welche in der dortigen Stadtbibliothek untergebracht ist: http://www.computerspielschule-greifswald.de

„Dein Spiel - dein Leben“ ist ein Projekt, welches jungen Gamern den verantwortungsvollen Umgang mit digitalen Welten näher bringen soll: http://dein-spiel-deinleben.de/

„Spielraum“ ist ein Institut zur Förderung der Medienkompetenz und ist an der FH Köln angesiedelt. Das Team hat eine Vielzahl an spannenden Projekten im Portfolio: http://www1.fh-koeln.de/spielraum/

„Spielkultur“ ist ein Blog von Electronic Arts, auf dem Sie viele spannende Informationen rund um die digitale Spielkultur bekommen: http://spielkultur.ea.de/

Das „Computerspielemuseum“ in Berlin ist immer eine Reise wert. Hier können Sie die Geschichte der Computergames hautnah erleben. Der Besuch des Computerspielemuseums ist genauso wichtig wie der Besuch der Gamescom, der Buchmesse oder eines Bibliothekartages: http://www.computerspielemuseum.de/

Ebenfalls empfehlenswert ist das Magazin „WASD - Texte über Games“. Hier geht es nicht um Rezensionen oder Ähnliches, sondern um die Auseinandersetzung mit vielen Facetten der Gameskultur: http://www.wasd-magazin.de/

Eine spannende Onlinedatenbank zu Games ist „Moby-Games“. Hier finden Sie zu beinahe jedem Computerspiel eine Vielzahl an spannenden Informationen: http:// www.mobygames.com/

Das Projekt „Internet Archive“ ermöglicht u.a. das Spielen von Retro-Games mittels Emulatoren: https://archive.org/

Der „Gamification Wiki“ ist eine Informationsplattform zum Thema Gamification. Hier gibt es viele spannende Beispiele und Konzepte rund um das Thema Gamification: http://gamification.org/

Ein ganz wichtiges Barcamp zur deutschen Spieleforschung ist „Researching Games“: http://researchinggames.net

Wie man Schulen durch Game-Mechaniken verbessern könnte, kann man auf der Seite von „Quest to Learn“ sehen. Diese öffentliche Schule aus den USA ist mit Sicherheit ein tolles Beispiel. Man kann auf der Seite einiges über die Inhalte erfahren und sich sogar den Lehrplan und andere Informationsmaterialien downloaden: http://q21.org/

Diese kleine Auswahl an Quellen zu weiteren Informationen ist natürlich nicht vollständig und sie ist zudem willkürlich ausgewählt. Auf meinem Blog und der Face- 
book-Gruppe von „games4culture“ werden Sie noch weitere Links finden. Dort werden die Links auch kontinuierlich geupdated.

Für Bibliotheken ist der „Tommi Softwarepreis“ ein ideales und erprobtes Projekt: http://www.kindersoftwarepreis.de/ 